\title{
Clues on post-Asymptotic Giant Branch Evolution and Planetary Nebulae Populations from the Magellanic Clouds.
}

\author{
Letizia Stanghellini \\ National Optical Astronomy Observatory 950 N. Cherry Ave., Tucson AZ 85719, USA
}

\begin{abstract}
.
The recent HST optical images, and the optical and ultraviolet spectra, of Magellanic planetary nebulae (PNe), together with the large data-base that has been collected in the past decade, allows unprecedented insight in the evolution of $\mathrm{PNe}$ and their central stars. In this paper we present a selection of recent results: The analysis of PN morphology, both in the optical and ultra-violet emission lines; the relation between nebular morphology and the chemistry produced by stellar evolution; the direct determination of the transition time from observations; and the study of the nature and evolutionary stage of the components of the planetary nebula luminosity function.
\end{abstract}

\section{Introduction}

Planetary nebulae (PNe) in the Magellanic Clouds (LMC, SMC) are the nearest extragalactic PNe known. They are close enough for detailed individual analysis, and they can be spatially resolved with the current technology; yet, they do not suffer the distance biases and extreme differential interstellar absorption that Galactic PNe are well known for. Magellanic PNe are thus the ideal probes to study stellar evolution of low- and intermediate-mass stars. They also are ideal benchmarks to extend the knowledge of PNe to the extra-Galactic PN populations, with the additional bonus of covering a large metallicity range.

PNe in the Magellanic Clouds have been observed from the ground, as point sources, since the sixties. The advent of the Hubble Space Telescope (HST) afforded their spatial resolution, making it possible to study their size, morphology, and their central stars (CSs). The work presented in this paper is based on the most recent $H S T$ data-sets acquired by the MCPN team ${ }^{1}$ in Cycles 8 through 10 with the Space Telescope Imaging Specrograph (STIS, Programs 8271, 8663, 9077, and 9120) and the Wide Field Planetary Camera 2 (WFPC2, Program 8702). Older archived narrow-band images (Program 6407, 5185, 4821, 4075, and 1266) were also used to augment the morphological data-set.

\footnotetext{
1 Team membership and other information are available in the MAST MCPN web page http://archive.stsci.edu/hst/mcpn/home/html
} 


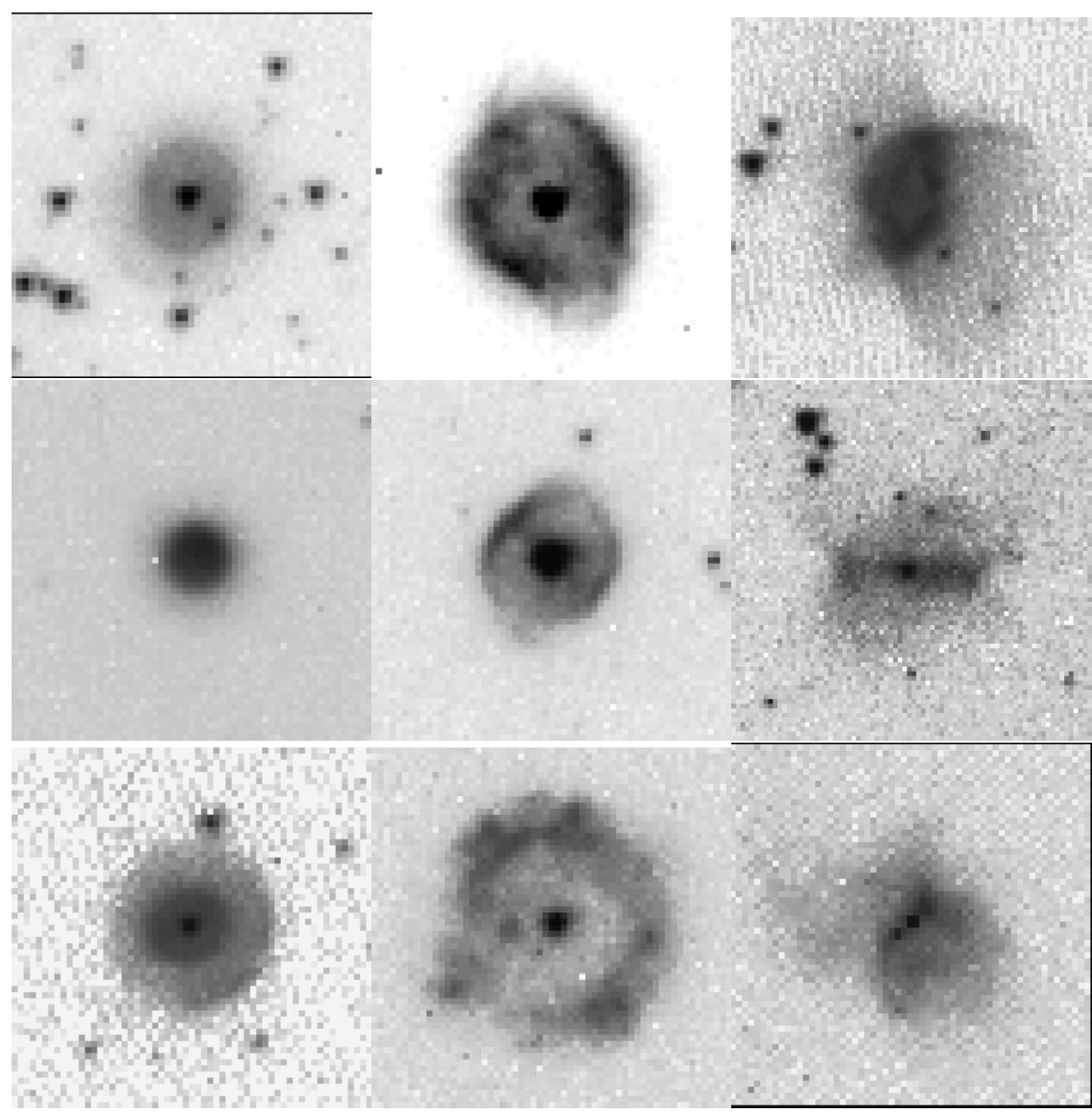

Fig. 1. Magellanic PNs. Left panel (each panel from top to bottom): R PNs LMC J 33, SMC SP 34, and LMC MG 40; Central panel: E PNs LMC SMP 101, SMC MG 8, and SMC MG 13; Right panel: B PNs LMC SMP 91, SMC MA 1682, and LMC MG 16. All thumbnails are $9 \operatorname{arcsec}^{2}$ sections of the STIS broad-band images

\section{Planetary Nebula Morphology}

The morphology of Galactic PNe has been studied thoroughly in the nineties, and it has been found that morphological type is related to the PN progenitor's evolutionary history and its mass. There is strong evidence that bipolar PNe are the progeny of the massive Asymptotic Giant Branch (AGB) stars (4-8 $\mathrm{M}_{\odot}$ ). Bipolar PNe are nitrogen-rich and carbon-poor $[1,2]$. The analysis of the morphological types and their distribution in a given PN population are then good indicators of age and evolutionary history.

Galactic PNe have been classified as round (R), elliptical (E), bipolar (B; includes quadrupolar and multipolar), bipolar core (BC; R or E PNe with a central bilobate concentration, or ring enhancement), and point-symmetric (P). A recent description of these classes is published by [1]. The majority of Galactic 
PNe are elliptical, but the actual number of B PNe could be underestimated, given that they typically lie in the Galactic plane (i.e., they may suffer from high reddening). PNe in the Magellanic Clouds, when spatially resolved, show the same admixture of morphological types than the Galactic PNe, as illustrated in Figure $1[3,4,5]$. While we do not attempt a statistical comparison of the Magellanic and Galactic PN morphological types, given the selection effects that hamper Galactic PNe, we can meaningfully compare the LMC and SMC samples. Both samples are characrerized by low field extinction, and they have been preselected in more or less the same way.

Table 1. Morphological Distribution

$\begin{array}{lrr}\text { Morphological type } & \text { \% LMC \% SMC } \\ & & \\ \text { Round (R) } & 29 & 35 \\ \text { Elliptical (E) } & 17 & 29 \\ \text { R \& E } & 46 & 64 \\ \text { Bipolar (B) } & 34 & 6 \\ \text { Bipolar core (BC) } & 17 & 24 \\ \text { Point-symmetric (P) } & 3 & 6\end{array}$

The results of the morphological distribution of PNe in the Clouds is illustrated in Table 1. One striking difference between the LMC and SMC morphological type distributions is that the fraction of B PNe in the LMC is almost six times that of the SMC. Furthermore, half of the LMC PNe are either bipolar or have a morphology that suggests a central ring (BC), while only a third of the SMC PNe fall in these two aspherical morphological classes.

From this analysis it results that the different processes involved in the formation of the different $\mathrm{PN}$ shapes occur in all galaxies where morphology has been studied. It is also apparent that the SMC environment may disfavor the onset of bipolarity in PNe. Otherwise, the different morphological statistics may indicate different populations of stellar progenitors in the two Clouds. While it seems reasonable to conclude that a low metallicity environment is unfavorable to bipolar formation and evolution, the exact causes have not been studied yet. A detailed study of metallicity and mass-loss may clarify this point. On the other hand, the different morphology counts may simply imply that most of the progenitors of the SMC PNe have masses in the lower-mass range $\left(\mathrm{M}<4 \mathrm{M}_{\odot}\right)$, thus they do not produce B PNe. A comparison of the SMC and LMC CS masses does not disagree with such a possibility [6].

\section{Results from UV Spectroscopy}

The study of the chemical composition that characterizes PN ejecta is a way to determine the origin and evolution of the progenitor stars. PNe contain the 


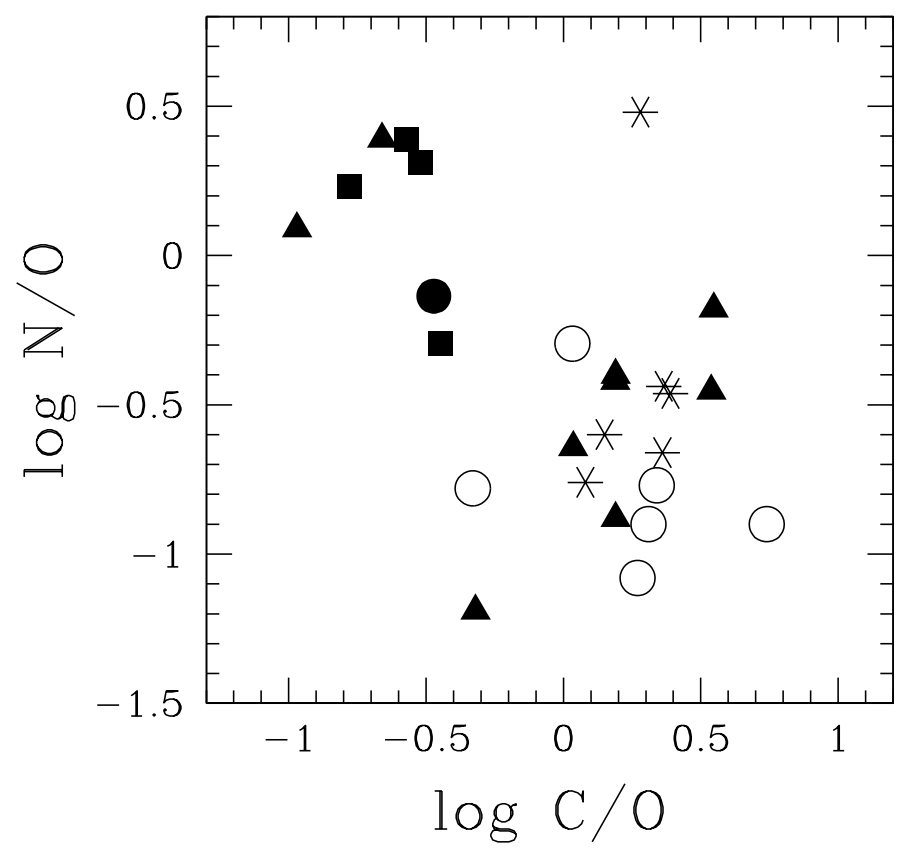

Fig. 2. The $\mathrm{C} / \mathrm{O}$ vs. N/O relation for $\mathrm{R}$ (open circles), E (asterisks), BC (triangles), $\mathrm{B}$ (squares), and $\mathrm{P}$ (filled circles) PNe in the LMC (adapted from [11])

products of stellar nucleosynthesis, dredged-up at the stellar envelope during the late AGB evolution. The study of carbon and nitrogen abundance in PNe is especially interesting. The theory of stellar evolution suggests that Galactic and Magellanic AGB evolution yield to different $\mathrm{N}$ and $\mathrm{C}$ concentrations depending on the AGB progenitor's mass. If $\mathrm{M}_{\mathrm{MS}}>4 \mathrm{M}_{\odot}$, the so-called hot-bottom burning (HBB) process would convert much of the carbon content into nitrogen, resulting in a different chemical make-up of the remnant PN [7]. In order to disclose possible correlation between the morphology and the evolutionary chemistry of the LMC PNe we need to have at our disposal the carbon and nitrogen abundance of a sufficient number of PNe to populate the major morphological classes. We use the nitrogen and oxygen abundances in the literature $[8,10]$ and 
the carbon abundance in $[9,11]$ to plot, in Figure 2, the relation between the (logarithmic) $\mathrm{C} / \mathrm{O}$ and $\mathrm{N} / \mathrm{O}$ abundances.

From this Figure we infer that the carbon stars progeny $(\log \mathrm{C} / \mathrm{O}>0)$ is limited to R, E, and BC PNe. B and P PNe originate from the evolution of stars that did not go though the carbon star phase, or, more likely, their progenitor underwent a carbon depletion, possibly due to the occurrence of the HBB. It appears that $\mathrm{R}$ and $\mathrm{E} \mathrm{PNe}$ are the progeny of lower-mass stars, those with $\mathrm{M}_{\mathrm{MS}}<4 \mathrm{M}_{\odot}$, while the opposite holds for the most aspherical (B and $\mathrm{P}$ ) PNe. From Figure 2 we see that BC PNe might have evolved from carbon stars in some cases, while in other cases they had evolved from more massive stars. It remains to be seen whether some of the BC PNe are indeed bipolar with faint lobes (those with low carbon and high nitrogen content), and others are misclassified $\mathrm{R}$ and $\mathrm{E}$ PNe.

In order to enlarge the sample of Magellanic PNe whose carbon abundance is known, we have used the HST data from program 9120, acquired with STIS grisms G140L and G230L [11]. The 2D spectra were obtained with a large aperture, showing the morphology of the PNe through the brightest UV emission line. It is then possible to compare the UV to optical morphology. In Figure 3 we show the clear optical images through the STIS CCD (left column) and the parts of the G230L spectra corresponding to the C III] emission line at $\lambda 1908$ $\AA$. The image sections are all to scale, thus the (relative) physical sizes are also to scale. The clear images have been rotated so their orientation coicides with the orientation of the 2D spectra. The UV emission deriving from the CIII] line originates in the same volume of the nebulae than the optical emission [4]. In Figure 3 we can also clearly see the spectrum of the central star of SMP 102.

The fitting of the UV stellar continua have been used to derive stellar tempeatures. In some cases, central stars of Magellanic PNe show P-Cygni profiles in the UV spectra [12].

\section{Stellar Evolution Beyond the AGB, and the Transition Time}

The known distance of Magellanic PNe makes them the ideal probes to study stellar evolution. AGB and post-AGB evolution have been studied theoretically and observationally in many different fashions. Nonetheless, there are still several processes that need to be understood to make the comparison between theory and data meaningful. In this section we describe how Magellanic PNe are used to shed some light on the transition time problem. Other aspect of AGB evolution will be examined in future papers.

When comparing CS data to theoretical predictions, it is often assumed that the time lag between the envelope ejection quenching (EEQ) and the PN illumination, the so-called transition time $\left(t_{t r}\right)$, has a duration either of a fixed value (generally $1000 \mathrm{yr}$ ), or it is set to zero. Both of these assumptions are inadequate to express a time-scale that is likely to assume different values in different PNe, and that represents a sizeable fraction of the PN life-time [13]. 


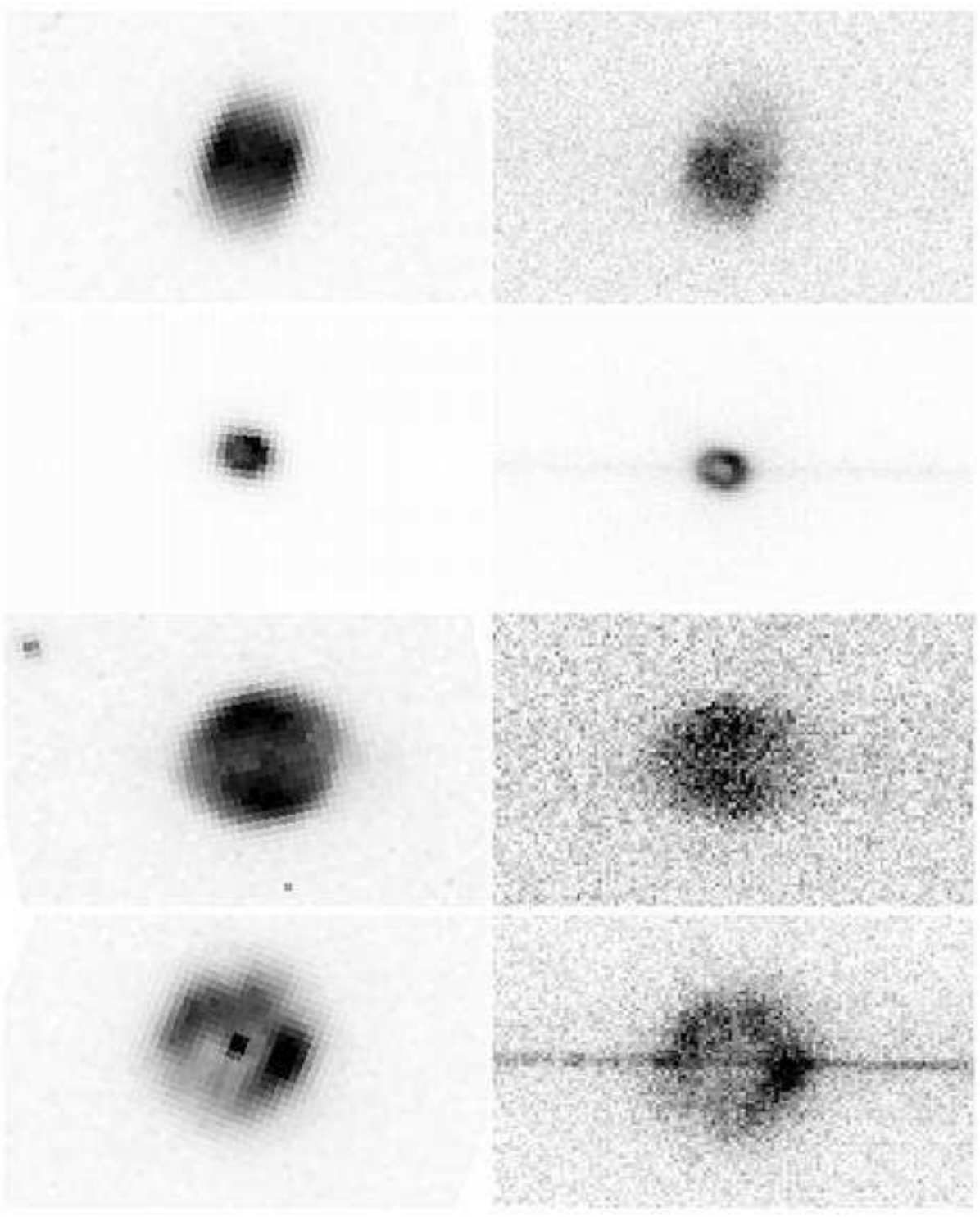

Fig. 3. Morphology of LMC PNe. Lerft column: clear STIS CCD images. Right column: C III] $1908 \AA$ images from the G230L 2D STIS spectra. The PNe are, from top to bottom, SMP 19, SMP 79, SMP 95, and SMP 102 

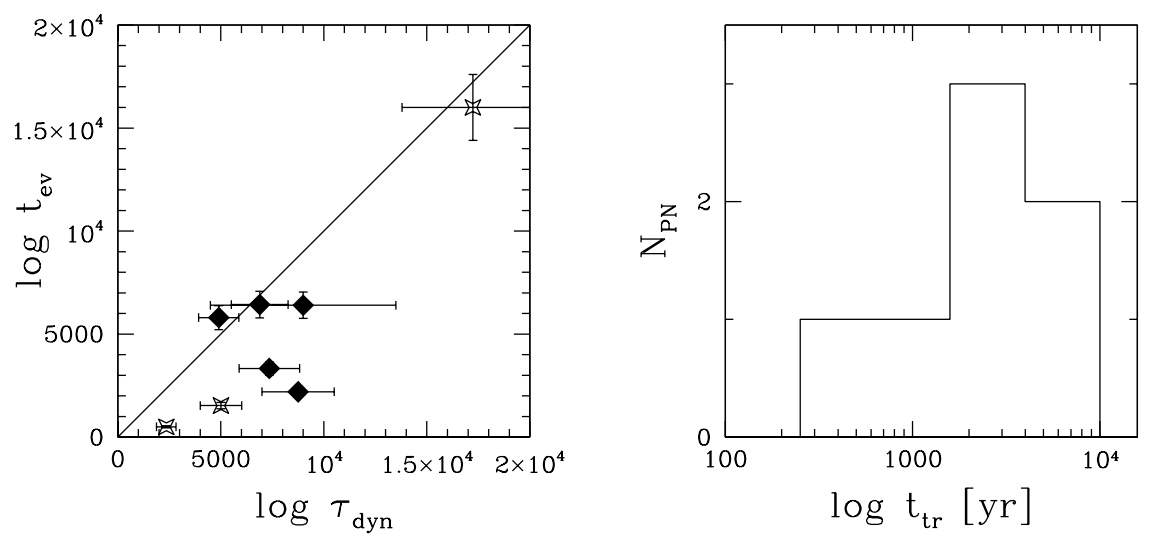

Fig. 4. (a): Evolutionary vs. dynamical time for LMC (diamonds) and SMC (stars) PNe. (b) : Transition time histogram

From a conceptual point of view, the transition time is mainly dependent on the residual envelope mass that survives the envelope ejection $\left(\mathrm{M}_{\mathrm{e}}^{\mathrm{R}}\right)$ and on the post-AGB mass-consumption process (or processes) that is in place in each specific CS. The dynamical time, $\tau_{\mathrm{dyn}}=D_{\mathrm{PN}} / v_{\mathrm{exp}}$ (where $\mathrm{D}_{\mathrm{PN}}$ is the diameter of the nebula and $\mathrm{v}_{\exp }$ is the nebular expansion velocity), represents the dynamical nebular age in the assumption that the nebular shell does not suffer acceleration. If we measure the diameter of the main nebular shell, the dynamical time tracks the evolutionary time measured from the EEQ event. In order to compare the dynamical time to the stellar evolutionary time we need to use the equation $\tau_{\mathrm{dyn}}=t_{\mathrm{tr}}+t_{\mathrm{ev}}$, where $\mathrm{t}_{\mathrm{ev}}$ is the evolutionary time evaluated after the PN illumination. This equation holds as long as the evolutionary tracks mark the temporal zero-point at $\mathrm{T}_{\text {eff }} \simeq 30,000 \mathrm{~K}$, as they usually do.

In order to estimate the transition time one needs to determine the residual envelope mass, the mass-loss during transition, and whether the CSs are burning hydrogen or helium. All these properties are rarely known, if ever. Another approach to determine, or at least constrain, the transition time is to use the Magellanic CSs. Since the distance to the Magellanic PNe is known, their CS mass can be estimated much more reliably than for Galactic PNe. In Figure 4 (a) we plot the evolutionary time of the Magellanic CSs, derived from their location in the HR diagram, against the dynamical time of their nebular shells. Stellar luminosities and temperatures used to deterine the CSs location on the HR diagram with respect to the Magellanic post-AGB evolutionary tracks are from [6] and [14]; the nebular diameters are from [4] and [5], and the expan- 
sion velocities are taken from [15]. Note that the majority of LMC post-AGB evolutionary tracks are He-burning tracks [16], while there is an indication that these stars are H-burners (see below in the PNLF section). To compensate for this mismatch we have scaled the $\mathrm{H}$ - versus He-burning tracks and increased the evolutionary time-scales accrodingly. Figure 4 (a) shows that the dynamical and evolutionary times correlate to one another $\left(\mathrm{R}_{\mathrm{xy}}=0.88\right)$, yet the dynamical time is usually higher, as predicted in most evolutionary cases [13]. There is one exception, LMC SMP 13, whose transition time is just below zero ( $t_{\mathrm{ev}}$ and $t_{d y n}$ are within their respective errorbars). From the evolutionary and dynamical times we derive the transition time in all cases but for SMP 13, whose transition time is obviously close to zero, or, alternatively, whose evolution occurs in thermal time-scale [13]. In Figure 4 (b) we report the histogram of the transition times for the Magellanic PNe whose dynamical and evolutionary times are available. The values of the transition time spread from a few hundreds to several thoudands years. In a few cases it goes to $10,000 \mathrm{yr}$, certainly a considerable fraction of the PN life-time. The most important conclusion from this exercise is that transition time can not be ignored when dealing with the evolution of $\mathrm{PNe}$ and their central stars. To our knowledge this is the first time that transition time has been effectively estimated for nebulae whose distance is reliable. A detailed analysis of the Magellanic PN estimated transition times and their models will be presented in a future paper.

\section{The Planetary Nebula Lumonsity Function}

The planetary nebula luminosity function (PNLF) is a well-known secondary distance indicator [17]. The high-luminosity cut-off of the PNLF seems to be invariant for galaxies with similar metallicites, while with a metallicity-correction it is also possible to unify the method for all galaxies. Despite its empirical success, it is still theoretically unclear why the PNLF is invariant for different PN population. Moreover, the evolutionary mechanisms behind the PNLF shape are still uncertain. In particular, the PNLF seems extremely hard to model. A realistic representation of the PNLF would involve several unknown and unconstrained parameters and processes related to the post-AGB and nebular evolution, such as the transition time, the mass-loss and its rates, the onset of PN morphology. The morphological data-base of Magellanic PNe may be used to gain insight in the PNLF. In Figures 5 and 6 we plot the luminosity of the LMC and the SMC $\mathrm{PNe}$ against their photometric physical radii. The luminosity is given in [O III] $\lambda 5007 \AA$ magnitudes, as typical for PNLFs, and the radii are in parsecs. All targets have been coded for morphological type, as described in the labels.

The snapshot survey targets plotted in Figures 5 and 6 were selected from the brightest LMC and SMC PNe [4, 5], thus the completeness near the high luminosity cut-off should be adequate [17]. In both Figures, but more clearly in Figure 5, one can see two different branches of PNe, running from small to large radii. These branches decline in brightness for larger radii, and the $\mathrm{B}$ and $\mathrm{P} \mathrm{PNe}$ populate the low brightness- large radii parts of the plots. The PNLF structure 


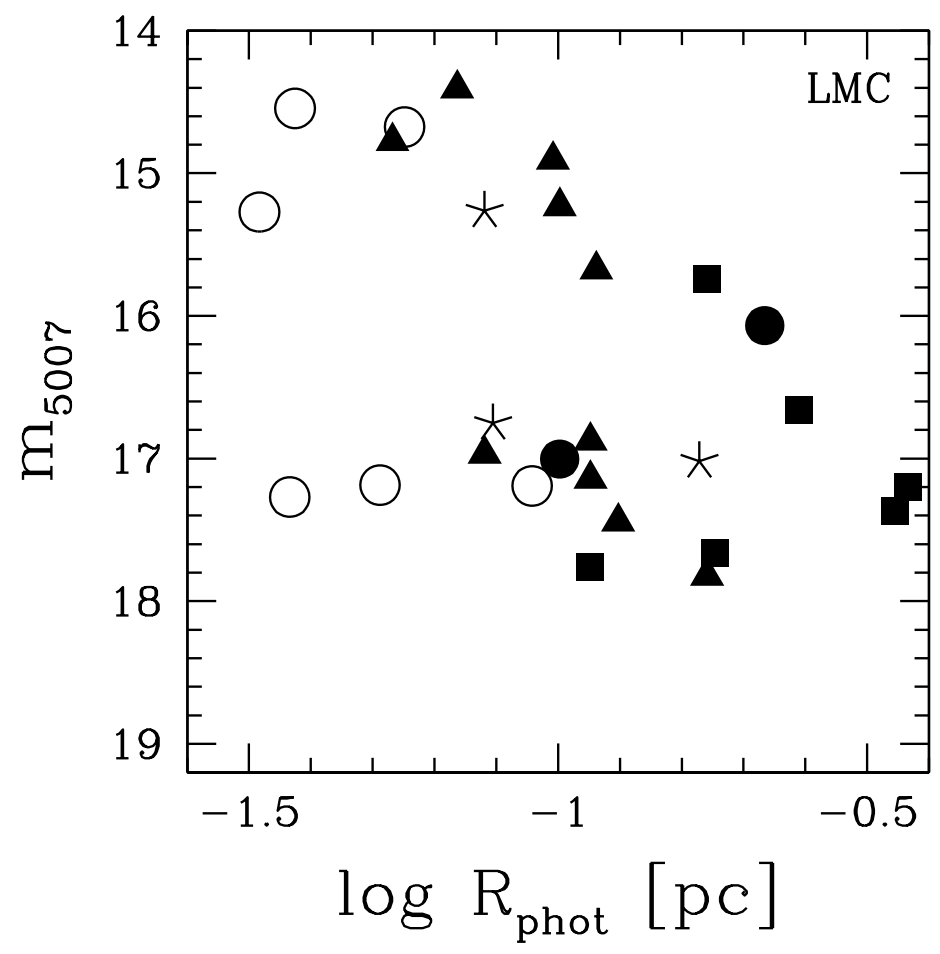

Fig. 5. Magnitude $\lambda 5007 \AA$ versus photometric radius for round (open circles), elliptical (asterisks), round and elliptical with a bipolar core (triangles), bipolar (squares), and point-symmetric (filled circles) PNe in the LMC 
that derives from the Figure has a dip at intermediate magnitudes, as predicted for H-burning post-AGB stars [13, 18].

More importantly, the high luminosity $\mathrm{PNe}$ in both Figures are R, E, and $\mathrm{BC}$. These are tipically the low nitrogen and high carbon PNe, the progeny of the lower mass stars $\left(\mathrm{M}<4 \mathrm{M}_{\odot}\right)$. The usefulness of this information is multifold: when modeling the high luminosity parts of the PNLF one could set aside the more difficult modeling of bipolar PNe, since spherical and ellipsoidal approximation should suffice. Furthermore, the mass range of $\mathrm{PN}$ progenitors that involve the high luminosity cut-off of the PNLF may be limited to $\mathrm{M}<4 \mathrm{M}_{\odot}$ in PNLF models.

The brighest PNe in the SMC are definitely R and E. The brightest B PNe are almost two magnitudes below the high luminsoity cut-off. In the LMC, there is a considerable fraction of bright BC PNe. Are these the same BC PNe with high carbon and low nitrogen abundances as seen in Figure 2? We have abundance information for three of the five bipolar core PNe in the brightest two magnitudes of the LMC PNLF, and these three objects have high carbon and low nitrogen content.

In Figure 6 we plot all the known (spatially-resolved) SMC PNe and the two H II regions corresponding to young stellar clusters, that were previously misclassified as PNe [19]. The Figure illustrated that misclassified PNe may occupy the same areas of the diagnostic $m_{5007}-\log R_{\text {phot }}$ plot. The brightest of these miscalssified PNe is just one magnitude below the high luminosity cut-off of the SMC PNLF. In a galaxy particularly rich of these ultra-compact H II regions that embedd young stellar clusters the distance determination based on the PNLF should be revisited after accounting for the contamination effects.

\section{Finale}

Magellanic PNe and their central stars have proven essential to probe postAGB evolution and the ensemble properties of $\mathrm{PNe}$, such as those related to the PNLF. The multi-wavelength data-base of Magellanic PNe is homogeneous, and now comprises spatially-resolved HST images of a represenaive fraction of $\mathrm{PNe}$ in these galaxies. This data-base allows umprecedented insight of stellar and nebulae evolution. In this paper we showed that R, E, B and P nebular morphology exists in both the LMC and the SMC, and that their relative frequency is different, being aspherical PNe rare in the SMC. Morphology in the light of the ultraviolet $\mathrm{C}$ III $] \lambda 5007 \AA$ line is similar to that of the major optical emission lines, such as $\mathrm{H} \alpha$ and [O III] $\lambda 5007 \AA$.

We have also shown that, as in the Milky Way, morphology correlates with nitrogen and carbon abundances, and that the aspherical PNe are typically carbonpoor and nitrogen-rich, as expected of the progeny of the high-mass AGB stars.

By comparing rate of nebular and stellar evolution of Magellanic PNe it has been possible to measure the time-lag between the EEQ and the PN illumination. The resulting transition time are a sizeable fraction of the nebular life-time 


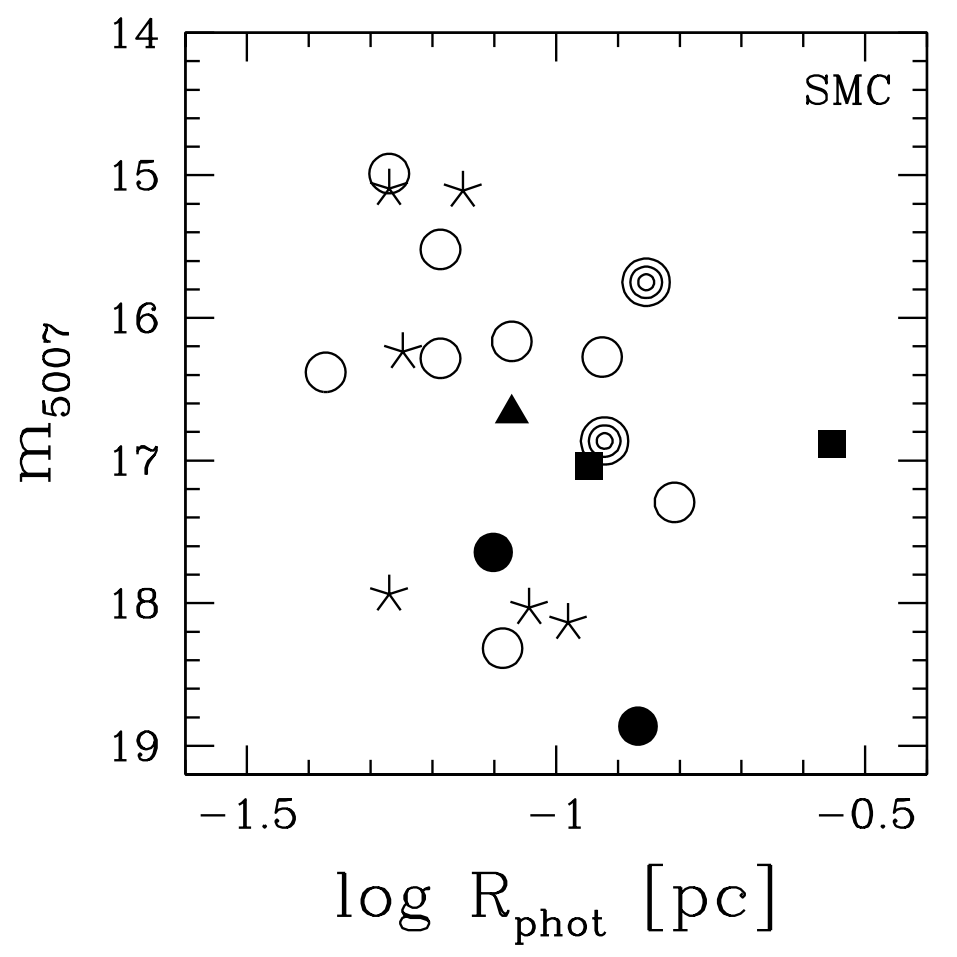

Fig. 6. Same as in Figure 5, for SMC PNe. The location of the target-like symbols correspond to two previously misclassified ultra-compact H II regions

in some cases. We conclude that the transition time can not be ignored when comparing nebular and stellar models.

The Magellanic PNLF can be studied in its components. The high luminosity $\mathrm{PNe}$ are generally $\mathrm{R}$ or $\mathrm{E}$, and, in all the cases where abundances have been calculated, the high luminosity $\mathrm{PNe}$ are carbon-rich and nitrogen-poor. This result has bearing on the future modeling of the PNLF.

Some of the results outlined here will be more sound when the planned Magellanic PN data-set will reach completion. In particular, we plan to obtain UV 
spectra of several SMC PNe to extend the result of PN carbon abundances to the SMC. The transition time calculation will be extended to the whole sample of Magellanic PNe whose central stars have been observed, and more SMC central stars will be observed as planned. Finally, a thorough study of the Magellanic PNLF is about to be completed by the MCPN team with the inclusion of the optical images form recent $H S T$ observations. The characteristics of the central stars hosted by the brightest nebulae in each galaxy will be studied in detail for more astrophysical insight in the invariance of the PNLF high luminosity cut-off across galaxies.

It is a pleasure to thank the MCPN team for their contribution to this project. Arturo Manchado and the IAC are warmly thanked for their hospitality in July 2004, when this paper was written.

\section{References}

1. L. Stanghellini, E. Villaver, A. Manchado, \& M. A. Guerrero: Ap. J. 576, 285 (2002)

2. M. Peimbert: IAU Symp. 76: Planetary Nebulae 76, ed. by Y. Terzian (Dordrecht: Reidel 1978) p. 215

3. L. Stanghellini, J. C. Blades, S. J. Osmer, M. J. Barlow, \& X.-W. Liu: Ap. J. 510, 687 (1999)

4. R. A. Shaw, L. Stanghellini, M. Mutchler, B. Balick, \& J. C. Blades: Ap. J. 548, 727 (2001)

5. L. Stanghellini, R. A. Shaw, B. Balick, M. Mutchler, J. C. Blades, \& E. Villaver: Ap. J. 596, 997 (2003)

6. E. Villaver, L. Stanghellini, \& R. A. Shaw: Ap. J. (in press)

7. L. B. van den Hoek \& M. A. T. Groenewegen: Astron. Astrophys. Suppl. Ser. 123, 305 (1997)

8. G. Stasinska, M. G. Richer, and M. L. McCall: Astron. Astrophys. 336, 667 (1998)

9. P. Leisy \& M. Dennefeld: Astron. Astrophys. Suppl. Ser. 116, 95 (1996)

10. P. Leisy \& M. Dennefeld: Astron. Astrophys. Suppl. Ser. (submitted)

11. L. Stanghellini, R. A. Shaw, \& D. Gilmore: Ap. J. (submitted)

12. A. Arrieta \& L. Stanghellini: (this volume)

13. L. Stanghellini \& A. Renzini: Ap. J. 542, 308 (2000)

14. E. Villaver, E., L. Stanghellini, \& R. A. Shaw: Ap. J. 597, 298 (2003)

15. M. A. Dopita, S. J. Meatheringham, B. L. Webster, \& H. C. Ford: Ap. J. 327, 639 (1988)

16. E. Vassiliadis \& P. R. Wood: Ap. J. Suppl. Ser. 92, 125 (1994)

17. R. Ciardullo: (this volume)

18. R. H. Mendez \& T. Soffner: Astron. Astrophys. 321, 898 (1997)

19. L. Stanghellini, E. Villaver, R. A. Shaw, \& M. Mutchler: Ap. J. 598, 1000 (2003) 